\title{
The effect of oxidation process on graphene oxide fiber properties
}

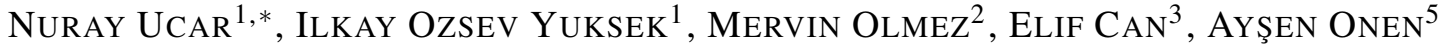 \\ ${ }^{1}$ Istanbul Technical University, Faculty of Textile Technologies and Design, \\ Department of Textile Engineering, Istanbul, Turkey \\ ${ }^{2}$ Istanbul Technical University, Department of Polymer Science and Technology, \\ Istanbul Technical University, Istanbul, Turkey \\ ${ }^{3}$ Istanbul Technical University, Department of Materials Science and Engineering, Istanbul, Turkey \\ ${ }^{4}$ Istanbul Technical University, Department of Chemistry, Istanbul, Turkey
}

\begin{abstract}
Graphene, a carbon allotrope, became a significant area of research with its superior electrical, mechanical, optical properties, etc. There are several methods to obtain graphene oxide from graphite, one of which is the Hummers method. In this study, several modifications and pre-treatments preceding the Hummers method have been employed. Three different graphene oxide fibers have been produced by three different procedures, i.e. fibers obtained by Hummers method with pre-oxidation step, modified Hummers method and modified Hummers method with pre-oxidation step. It has been observed that pre-oxidation has a significant effect on graphene oxide fiber properties produced by wet spinning process (coagulation). Modified Hummers method without pre-oxidation leads to the highest breaking strength and breaking elongation. Reduced fiber linear density, breaking strength and breaking elongation together with increased crimp were observed in graphene fiber due to the addition of pre-oxidation step.
\end{abstract}

Keywords: pre-oxidation; graphene oxide fiber; Hummers method; wet spinning; coagulation

\section{Introduction}

An increasing demand on superior properties of materials led to development of new materials. As a result, applications of carbon and carbon based structures such as two-dimensional graphene have gained high importance, recently. Pristine graphene cannot be processed directly, it is converted into graphite oxide for mass production. Brodie, in 1859 , developed the first method to form graphite oxide from graphite using for this purpose potassium chlorate $\left(\mathrm{KClO}_{3}\right)$, graphite and fuming nitric acid [1]. Staudenmaier further improved this method in 1898 by adding sulfuric acid to the reaction [2]. Hummers, developed an alternative method in 1958, in which sodium nitrate, sulfuric acid and potassium permanganate were used in the reaction [3]. Since then, researchers implemented Hummers method, which is known as relatively

*E-mail: ucarnuray@gmail.com safe, and simple compared to the methods previously developed. Up to now, the Hummers method has been modified for improved safety and ease of processing. $\mathrm{Wu}$ and Ting [4] varied synthesis conditions of Hummers method to obtain GO. The concentrations of the chemicals involved, and addition rates of those chemicals along with water have been changed. Higher yields were observed with the reduction of water dropping rate. Additionally, the $\mathrm{NaNO}_{3} / \mathrm{KMnO}_{4}$ ratio was found as an important factor that affects the yield. According to the XRD and Raman spectroscopy results, no obvious effect of the synthesis conditions (except for the size of graphene oxide particles) was observed. The procedure applied by Wu et al. [4] was also studied by Ali et al. [5]. In the research by Ali et al., the properties of two different modified Hummers methods including the method applied by Wu et al. [4], and so called inter-step stirring method, were compared. In the inter-step stirring method, each consequent step in the modified 
Hummers method was followed by stirring. The inter-stirring method produced low thickness (more than $38 \%$ less) GO sheets at $2.46 \%$ higher yield. Ardakani et al. [6] examined the result of modified Hummers method by using centrifugation instead of filtration to exfoliate graphite. The centrifuge was set to $6000 \mathrm{rpm}$ for $10 \mathrm{~min}$, and $\mathrm{HCl}$ was used. According to the EDS some residuals remained after the process. Additionally, enhanced quality was observed with increased number of washings connected with centrifugations. Chen et al. [7] studied an environmental friendly approach, consisting in eliminating $\mathrm{NaNO}_{3}$ in Hummers method. The GO sheets obtained by Hummers and newly introduced modified method were similar in terms of chemical structure and properties such as lateral dimensions and dispersion behavior. Paulchamy et al. [8] used a modified Hummers method in which thermal treatment was employed in both exfoliation and oxidation steps. The exfoliation, and formation of graphene sheets were proved by SEM and FE-SEM microscopy. In another study by Marcano et al. [9], an improved Hummers method was adapted. The method was conducted with $\mathrm{KMnO}_{4}$, $\mathrm{NaNO}_{3}, \mathrm{H}_{2} \mathrm{SO}_{4}$. Use of $\mathrm{NaNO}_{3}$ was eliminated along with the use of excess amounts of $\mathrm{KMnO}_{4}$. To obtain the oxidation process with better efficiency, the ratio of $\mathrm{H}_{2} \mathrm{SO}_{4}$ to $\mathrm{H}_{3} \mathrm{PO}_{4}$ was selected as 9:1. The improved method was found to be more ecological. It was observed that there was no toxic gas formed, and increased regularity of the graphene oxide was observed.

All of these methods have been developed to obtain graphene oxide (GO) particles from graphite, and those methods focused on examining the effect of production parameters on final graphene oxide particle properties without conversion into GO fiber. In this study, for the first time, the authors investigated the effect of oxidation methods (Hummers, modified Hummers and pre-oxidized modified Hummers methods) on resultant GO fiber properties, while the studies performed previously focused on determining the effect of processing conditions such as nozzle size, feed rate and reduction time with Vitamin $\mathrm{C}$, and the effect of coagulation time, number of coagulation baths and ingredients on resultant graphene oxide fiber properties [10, 11].

\section{Experimental}

\subsection{Materials}

Expandable graphite (30 $\mu \mathrm{m}$ thickness, $300 \mu \mathrm{m}$ diameter and $99 \%$ purity) was purchased from Grafen Co. $\mathrm{KMnO}_{4}$ (potassium permanganate), $\mathrm{H}_{2} \mathrm{SO}_{4}$ (sulfuric acid), $\mathrm{K}_{2} \mathrm{O}_{8} \mathrm{~S}_{2}$ (potassium persulfate), $\mathrm{P}_{2} \mathrm{O}_{5}$ (phosphorus pentoxide), $\mathrm{HCl}$ (hydrochloric acid), $\mathrm{CaCl}_{2}$ (granular calcium chloride) and ethanol were obtained from Merck. $\mathrm{NaNO}_{3}$ (sodium nitrate) was supplied from $\mathrm{ZAG}$ and $\mathrm{H}_{2} \mathrm{O}_{2}$ (hydrogen peroxide) was purchased from Carlo Erba.

\subsection{Methods}

Three different methods have been applied to obtain graphene oxide from graphite. The first one was Hummers method with pre-oxidation step symbolized as PH. In the second method, Hummers method was modified; it was described as Modified Hummers method with pre-oxidation step and symbolized as PMH. In the third method, modified Hummers method was applied without pre-oxidation and symbolized as $\mathrm{MH}$.

\subsubsection{Hummers method with pre-oxidation step (PH)}

The amounts of graphite per sulfuric acid (graphite: $\mathrm{H}_{2} \mathrm{SO}_{4}$ ), graphite per potassium persulfate (graphite: $\mathrm{K}_{2} \mathrm{O}_{8} \mathrm{~S}_{2}$ ) and graphite per phosphorus pentoxide (graphite: $\mathrm{P}_{2} \mathrm{O}_{5}$ ) were selected as $1: 60,1: 0.84$ and $1: 1.24$ by mass respectively.

Graphene - GIC and $\mathrm{H}_{2} \mathrm{SO}_{4}$ were stirred with a magnetic stirrer which was set to $80{ }^{\circ} \mathrm{C}$. When the temperature reached $50{ }^{\circ} \mathrm{C}, \mathrm{K}_{2} \mathrm{O}_{8} \mathrm{~S}_{2}$ and $\mathrm{P}_{2} \mathrm{O}_{5}$ were added carefully. The timer was set to 5 hours for continuous stirring as soon as the temperature of the mixture reached $80{ }^{\circ} \mathrm{C}$. After being cooled, distilled water was added carefully. The mixture was left to stand for 24 hours. The liquids in the mixture were decanted and the remained dry phase was separated equally into centrifuge tubes. In the centrifuge, several washings were performed 
at $5500 \mathrm{rpm}$ with the use of DI water. When $\mathrm{pH}$ level reached 5 to 6 , the pre-oxidized graphene particles remainding at the bottom were left to dry. Afterwards, graphite oxide was produced according to the Hummers method [10]. In this method, preoxidized graphite with $\mathrm{NaNO}_{3}$ and $\mathrm{H}_{2} \mathrm{SO}_{4}$ were stirred uniformly in an ice bath with a magnetic stirrer for $10 \mathrm{~min}$. Afterwards, $\mathrm{KMnO}_{4}$ was added slowly and carefully to the mixture. After several heating, cooling processes, the mixture diluted by distilled water was set aside for $24 \mathrm{~h}$, then hydrogen peroxide $\left(\mathrm{H}_{2} \mathrm{O}_{2}\right)$ was added to the mixture in order to stop the reaction. The color of solution was changed into yellowish. Graphene oxide dispersion was washed with $1 \mathrm{M} \mathrm{HCl}$ solution to remove impurities. Then, the graphene oxide dispersion was centrifuged by distilled water in the Nüve, NF 800R centrifuge, until the $\mathrm{pH}$ reached $\mathrm{pH} 5$ to 6.

\subsubsection{Modified Hummers method with pre- oxidation step (PMH)}

Similar method as described in "Hummers method with pre-oxidation step (PH)" has been applied except for diluting with distilled water and setting aside for 24 hours during Hummers method. Distilled water has been added into graphite mixture treated with $\mathrm{KMnO}_{4}$ and it was kept for 5 day instead of 24 hours, in order to get strong reaction. Then, hydrogen peroxide $\left(\mathrm{H}_{2} \mathrm{O}_{2}\right)$ was added to the mixture in order to stop the reaction and all remaining processes described in "Hummers method with pre-oxidation step $(\mathrm{PH})$ " were applied as for the remaining fibers.

\subsubsection{Modified Hummers method (MH)}

Similar method as described in "Modified Hummers method with pre-oxidation step (PMH)" has been applied except for pre-oxidation process. Without applying pre-oxidation, the Modified Hummers method has been applied to graphite.

\subsection{Graphene oxide fiber production from graphene oxide dispersion}

The prepared graphene oxide was dispersed by mechanical homogenizer (WiseTis Homogenizer, HG-15D), at $1000 \mathrm{rpm}$ for 90 minutes.
The graphene oxide fibers were manufactured by processing graphene oxide dispersion through 3 coagulation baths with a rate of $20 \mathrm{mg} / \mathrm{mL}$ by using a laboratory syringe system. The graphene oxide fibers were produced through a wet spinning process (coagulation method) by using a nozzle (19 gauge needle, inner diameter: $0.69 \mathrm{~mm}$ ).

The amounts of the ingredients of the first, second and third coagulation baths are shown in Table 1.

During the fiber production process from graphene oxide dispersion, $\mathrm{CaCl}_{2}$ was added into the first coagulation bath due to its ionic crosslinking properties. The $\mathrm{Ca}^{+2}$ ions and ethanol were absorbed during desorption of water, and $\mathrm{Ca}^{+2}$ ions bonded the graphene flakes to each other while supporting the fiber formation process. After third coagulation bath, GO fiber was dried in laboratory conditions. During drying, a decrease in fiber diameter along with increased surface crimp was observed as a result of evaporation of water and ethanol. From previous FT-IR studies done on Modified Hummers samples, it was observed that the $\mathrm{O}-\mathrm{H}$ hydroxyl pick $\left(3200 \mathrm{~cm}^{-1}\right.$ to $3300 \mathrm{~cm}^{-1}$ ) intensity was reduced when GO dispersion was converted into GO fiber due to evaporation of water. Additionally, $\mathrm{C}=\mathrm{O}$ carboxyl pick $\left(1720.27 \mathrm{~cm}^{-1}\right)$ disappeared when GO dispersion was converted into GO fiber. this might be due to the reaction between $\mathrm{Ca}^{+2}$ ions and $\mathrm{C}=\mathrm{O}$ groups after coagulation.

Table 1. Ingredients of coagulation baths.

\begin{tabular}{cccc}
\hline $\begin{array}{c}\text { Coagulation } \\
\text { Bath } \\
\text { Number }\end{array}$ & $\begin{array}{c}\text { Ethanol } \\
{[\mathrm{ml}]}\end{array}$ & $\begin{array}{c}\text { DI water } \\
{[\mathrm{ml}]}\end{array}$ & $\begin{array}{c}\mathrm{CaCl}_{2} \\
{[\mathrm{~g}]}\end{array}$ \\
\hline \hline 1 & 30 & 70 & 5 \\
2 & 40 & 60 & - \\
3 & 50 & 50 & - \\
\hline
\end{tabular}

The schematic of the wet spinning process is shown in Fig. 2. The abbreviations of fibers obtained are as follows:

PH: Preoxidation based on Hummers method (reaction for 1 day with preoxidation) 


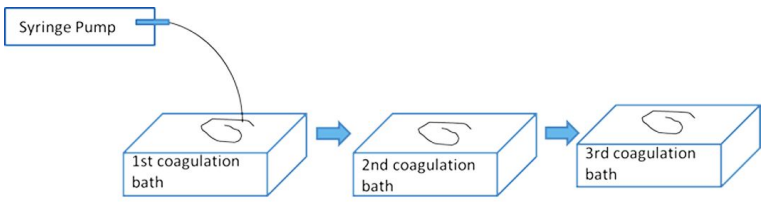

Fig. 1. Wet spinning process

PMH: Preoxidation based on modified Hummers method (reaction for 5 days with preoxidation).

MH: Modified Hummers method (reaction for 5 days without preoxidation).

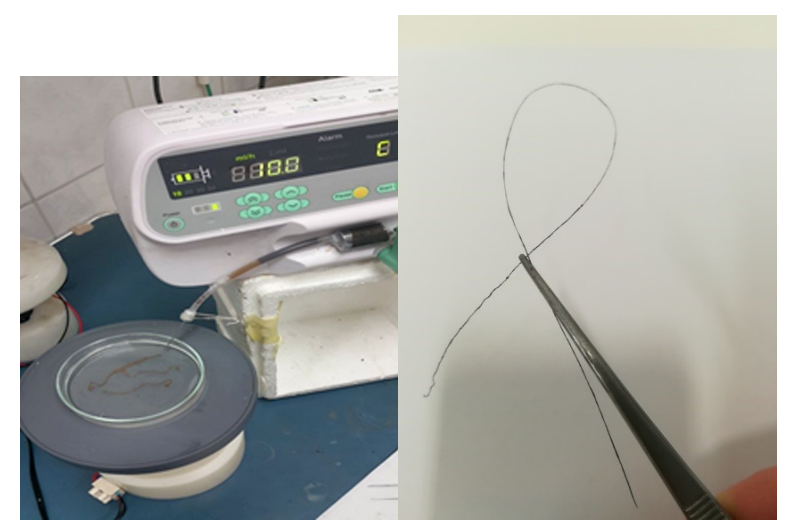

(a)

(b)

Fig. 2. (a) The wet spinning process; b) Wet-spun fiber

\section{Measurements and characterization}

Characterization of GO fibers for surface morphology: scanning electron microscopy (SEM, QUANTA FEG 200) in ESEM (Environmental SEM) mode was used for morphological analyses. Samples coated with $\mathrm{Au} / \mathrm{Pd}$ by sputtering (Quorum, SC7620) under vacuum conditions (2 $\mathrm{Pa}$ ) were examined under ESEM.

Characterization of $G O$ fibers for mechanical properties: Mechanical properties of the fibers were measured by using Usel UNF 15 tensile tester according to the ASTM D3822-07 standard. Crosshead speed was set to $1 \mathrm{~mm} /$ minute and the gauge length was set to $10 \mathrm{~mm}$. Average value of more than 10 measurements were taken for each sample.

Characterization of $G O$ fibers for electrical conductivity: The conductivities $(\mathrm{S} / \mathrm{cm})$ of the fibers were calculated depending on the electrical resistance values measured by $\mathrm{Mi}$ crotest 6370 LCR meter with a two probe. Average value of more than 10 measurements was obtained for each sample. Following equation has been used to calculate the electrical conductivity coefficient:

$$
\rho=L /(A * R)
$$

where $\rho$ is a coefficient of electrical conductivity $(\mathrm{S} / \mathrm{cm}), \mathrm{R}$ is electrical resistance $(\mathrm{ohm}), \mathrm{A}$ is a cross sectional area $\left(\mathrm{cm}^{2}\right), \mathrm{L}$ is length $(\mathrm{cm})$.

Characterization of $G O$ fibers with $X$-ray photoelectron spectroscopy (XPS): The XPS analysis was performed with Thermo Scientific K-Alpha X-ray photoelectron spectrometer. The XPS analyses were conducted on samples before fiber formation, right after finishing the production process using the Hummers method. The binding energies were arranged depending on the $\mathrm{C} 1 \mathrm{~s}$ lines and $\mathrm{k}$-alpha radiation was used for recording the XPS spectra. The energy step size was $0.1 \mathrm{eV} \cdot \mathrm{s}^{-1}$ , and the pass energy was set to $150 \mathrm{eV}$. The pressure in the analysis chamber was approximately $8 \times 10^{-3} \mathrm{~Pa}$. For data analysis, peak intensities were arranged by determination of Shirley-tip background, arrangement of the lines for Lorentz (30\%) and Gauss (70\%) line combination and integration of all peak points.

\section{Results and discussion}

Surface Morphology Characterization of GO Fibers: As can be seen in Fig. 3, the fibers obtained from modified Hummers ( $\mathrm{MH})$ and pre-oxidized modified Hummers methods (PMH) had smoother surfaces compared to the fiber obtained from preoxidized Hummers (PH) method. As is known, collapsed and porous structures along with reduced mechanical performance are observed when rapid coagulation takes place [12]. High surface crimp of PH fiber observed in SEM images might be due to sudden, rapid and increased coagulation, i.e. leaving more water from GO dispersion suddenly into coagulation bath. Furthermore, less reaction time (24 hour) for fibers obtained with PH results in less functional groups on the flake surface which is also confirmed by XPS spectroscopy. Less functional 
groups may lead to collapsed, porous and crimpy structure, since reduction studies which have been performed to reduce functional groups pointed out that an increase of reduction time results in more crimped surface structure $[13,14]$.
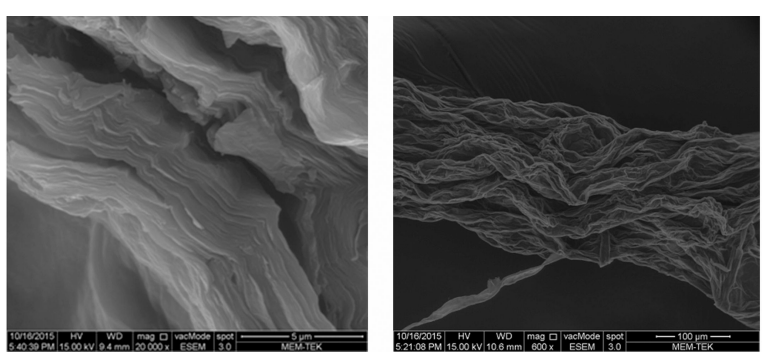

(a)
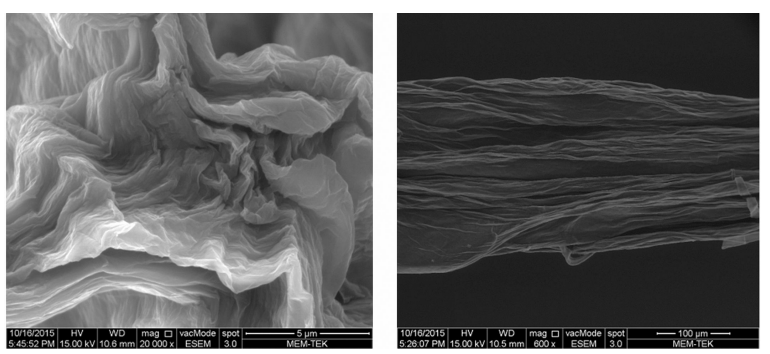

(b)
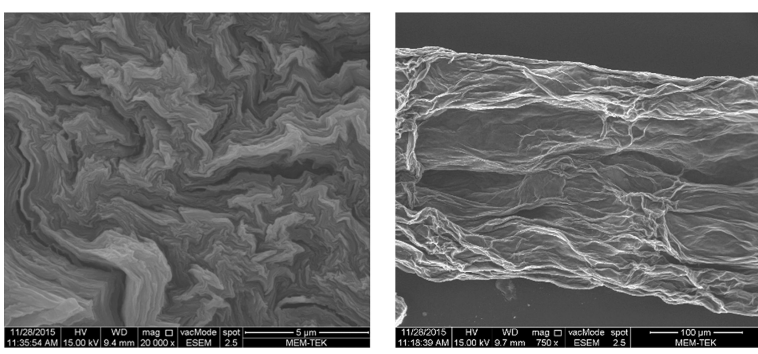

(c)

Fig. 3. SEM images of GO fibers: (a) fiber obtained by pre-oxidized Hummers method $(\mathrm{PH})$, (cross sectional image and longitudinal image respectively); (b) fiber obtained by modified Hummers method (MH), (cross sectional image and longitudinal image respectively); (c) fiber obtained by modified pre-oxidized Hummers method $(\mathrm{PMH})$, (cross sectional image and longitudinal image respectively).

GO fiber linear density The fiber linear density (Tex), which is the weight in grams of 1000 meters of GO fibers, for three prepared samples are shown in Table 2.
Table 2. Fiber linear densities

\begin{tabular}{cc}
\hline Sample & $\begin{array}{c}\text { Fiber Linear } \\
\text { Density (tex) }\end{array}$ \\
\hline \hline PH $(\mathrm{KI}-20 \mathrm{~mL} / \mathrm{h})$ & 23.7 \\
$\mathrm{MH}(\mathrm{KI}-20 \mathrm{~mL} / \mathrm{h})$ & 25.4 \\
$\mathrm{PMH}(\mathrm{KI}-20 \mathrm{~mL} / \mathrm{h})$ & 22.5 \\
\hline
\end{tabular}

As can be seen in Table 2, the fiber linear density of the fibers produced with $\mathrm{MH}$ is the highest, and it decreased when the pre-oxidation step was applied. Pre-oxidation process may lead to deterioration of graphene flakes, resulting in smaller graphene flake diameter and mass loss [13-15]. By using the modified Hummers method, the functional groups increased as can be seen from the XPS spectroscopy results which may lead to an increase of weight with less material loss than that observed for pre-oxidized samples.

Mechanical properties of GO fibers The mechanical properties of GO fibers are shown in Table 3.

Table 3. Mechanical Properties of GO fibers

\begin{tabular}{cccc}
\hline Sample & Values & $\begin{array}{c}\text { Breaking } \\
\text { elongation } \\
{[\%]}\end{array}$ & $\begin{array}{c}\text { Breaking } \\
\text { strength } \\
{\left[\mathrm{N} / \mathrm{mm}^{2}\right]}\end{array}$ \\
\hline \hline \multirow{2}{*}{$\mathrm{PH}$} & Average & 4.41 & 21.75 \\
& $\% \mathrm{CV}$ & 26.92 & 29.56 \\
\hline \multirow{2}{*}{$\mathrm{MH}$} & Average & 5.43 & 37.21 \\
& \% CV & 29.33 & 29.89 \\
\hline \multirow{2}{*}{ PMH } & Average & 3.22 & 31.64 \\
& $\% \mathrm{CV}$ & 21.27 & 29.77 \\
\hline
\end{tabular}

As can be seen in the Table 3, the highest breaking strength and elongation were observed in the fibers produced with MH method. The oxidation process increased the functional groups and changed the graphene morphology that might cause two opposite effects. Functional groups increased with the oxidation process as can be seen from XPS spectra. Increased functional groups may lead to more homogenous dispersion and formation of stronger bonds between graphene flakes which resulted in enhanced mechanical properties. 
On the other hand, pre-oxidation process may lead to formation of smaller graphene flakes and material loss [13-15], resulting in lower breaking strength and breaking elongations.

Electrical conductivity of GO fibers

The electrical conductivities of the fibers are given in Table 4. As can be seen from the table, there is not a clear difference in the electrical conductivities. All fibers produced are in the semiconductor range. Ucar et al observed that the electrical conductivity increased from $8.88 \times 10^{-4}$ to 4.80 when 2.5 hours of Vitamin $\mathrm{C}$ reduction was followed, and raised to 6.18 after 5 hours of reduction time [14].

Table 4. Electrical conductivities of GO fibers

\begin{tabular}{ccc}
\hline Sample & $\begin{array}{c}\text { Average electrical } \\
\text { conductivity }[\text { S/cm] }]\end{array}$ & $\begin{array}{c}\mathrm{CV} \\
{[\%]}\end{array}$ \\
\hline \hline $\mathrm{PH}$ & $2.9 \times 10^{-4}$ & 29.6 \\
$\mathrm{MH}$ & $4.9 \times 10^{-4}$ & 29.9 \\
$\mathrm{PMH}$ & $8.1 \times 10^{-4}$ & 6.82 \\
\hline
\end{tabular}

$\underline{X \text {-ray photoelectron spectroscopy (XPS) results of }}$ GO fibers

The $\mathrm{C}$ and $\mathrm{O}_{2}$ contents in the samples after $\mathrm{X}$-ray photoelectron spectra are shown in Table 5 and Fig. 4. As can be seen from Table 5, fibers manufactured by PMH method contain more functional groups than fibers produced by $\mathrm{MH}$ method as a result of the peroxidation step. Oppositely, the fibers produced by $\mathrm{MH}$ contain more functional groups compared to fibers obtained by $\mathrm{PH}$. This is due to the five-day oxidation step performed during the modified Hummers process, and increased number of functional groups as a result of the oxidation process.

\section{Conclusions}

This study contributes to the current literature by comparing the resultant graphene oxide fiber properties obtained from different oxidation process such as Hummers method $(\mathrm{PH})$ with preoxidation step, modified Hummers'method (MH) and modified Hummers method with pre-oxidation step (PMH). Following results have been obtained:
Table 5. The $\mathrm{C}$ and $\mathrm{O} 2$ content and binding energies of the samples after $\mathrm{X}$ - ray photoelectron spectra

\begin{tabular}{cccc}
\hline Sample & $\mathrm{C} / \mathrm{O}$ & $\begin{array}{c}\mathrm{C} 1 \mathrm{~s} \\
\mathrm{BE}[\mathrm{eV}]\end{array}$ & $\begin{array}{c}\mathrm{O} 1 \mathrm{~s} \\
\mathrm{BE}[\mathrm{eV}]\end{array}$ \\
\hline PH & 2.38 & 285.0 & 533 \\
$\mathrm{MH}$ & 2.22 & 285.0 & 533.09 \\
$\mathrm{PMH}$ & 2.17 & 285.0 & 533.06 \\
\hline
\end{tabular}

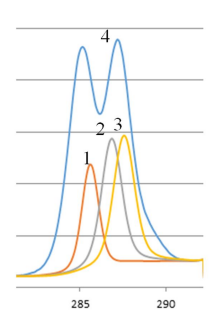

PH

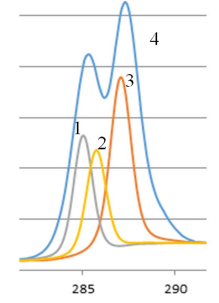

$\mathrm{MH}$

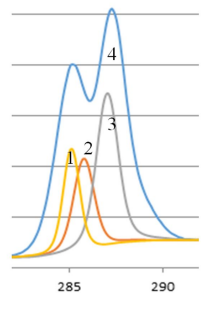

PMH
Fig. 4. X- ray photoelectron spectra of $\mathrm{PH}, \mathrm{MH}$ and $\mathrm{PMH}$ samples representing the binding energies of $\mathrm{C}$ and $\mathrm{O}_{2}$ bonds, 1: Peak 1, 2: Peak 2, 3: Peak 3, 4: Sum of peaks; a) PH; b) MH; c) PMH

- Smooth fibers can be obtained with the elimination of pre-oxidation step.

- Pre-oxidation step resulted in reduction of fiber linear density and crinkly surface. The reduction of fiber linear density might be due to the reduction of graphene flake diameter and material loss due to the preoxidation process.

- Longer oxidation duration during Hummers process in modified Hummers method increased the functional groups in the structure, and pre-oxidation incorporated modified Hummers method led to increase of functional groups.

- Pre-oxidation step might also cause deterioration of graphene flakes which resulted in decrease of breaking strength.

- There was no clear difference in electrical conductivities of the fibers due to the oxidation, all fibers were in semi-conductor range.

\section{Acknowledgements}

This study is supported by TUBITAK (Project Number 114M524). 
Table 6. Binding energies of samples

\begin{tabular}{|c|c|c|c|c|c|c|}
\hline & \multicolumn{2}{|c|}{$\begin{array}{c}\text { Peak } 1 \\
\text { C-C, C-H }\end{array}$} & \multicolumn{2}{|c|}{$\begin{array}{c}\text { Peak } 2 \\
-\mathrm{C}-\mathrm{OH},-\mathrm{C}-\mathrm{OR}\end{array}$} & \multicolumn{2}{|c|}{$\begin{array}{l}\text { Peak } 3 \\
-\mathrm{C}=\mathrm{O}\end{array}$} \\
\hline & $\begin{array}{l}\mathrm{BE} \\
{[\mathrm{eV}]} \\
\end{array}$ & [\%] & $\begin{array}{c}\mathrm{BE} \\
{[\mathrm{eV}]} \\
\end{array}$ & {$[\%]$} & $\begin{array}{c}\mathrm{BE} \\
{[\mathrm{eV}]} \\
\end{array}$ & {$[\%]$} \\
\hline $\mathrm{PH}$ & 285.59 & 16.2 & 286.84 & 23.92 & 287.56 & 24.65 \\
\hline $\mathrm{MH}$ & 285.02 & 19.9 & 285.73 & 17.56 & 287.05 & 30.76 \\
\hline PMH & 285.12 & 14.71 & 285.79 & 16.19 & 287.04 & 28.43 \\
\hline
\end{tabular}

\section{References}

[1] Brodie B. C., Philos. Trans. R. Soc. London, 149 (1859), 249.

[2] Staudenmaier L., Ber. Dtsch. Chem. Ges., 31 (1898), 1481.

[3] Hummers W.S., Offeman R.E., J. Am. Chem. Soc., 80 (6) (1958), 1339.

[4] Wu T. T. And Ting J. M., Surf. Coat. Technol., 231 (2013), 487.

[5] Ali A., Jamil M., Khan Z. S., Ahmed S., Habib A., Ahmad N., Power Generation System and Renewable Energy Technologies (PGSRET), (2015).

[6] Ardakani S.E.M., Singh B.S.M., Mohammed N.M., AIP Conference Proceedings, (2015), 1669.

[7] Chen T., Zeng B., LiU J.L., Dong J.H., LiU X.Q., Wu Z., YANG X.Z., Li Z.M., $8^{\text {th }}$ China International Nanoscience and Technology Symposium (CINSTSO9) IOP Publishing Journal of Physics: Conference Series, (2009), 188.

[8] Paulchamy B., Arthi G., Lignesh B.D., $J$. Nanomed. Nanotechnol., 6 (2015), 1.
[9] Marcano D.C., Kosynkin D. V., Berlin J. M. Sinitskit A., Sun Z., Slesarev A., Alemanyi L.B., Lu W., Tour J.M., ACS Nano, 4 (8) (2010), 4806.

[10] Ucar N., Gokceli G., Onen A., Yuksek I.O., KARATEPE YAVuZ N., J. Indian. Tex., 2016

[11] UCAR N., Gokceli G., ONEN A., YuKsek I.O., KaratePe Yavuz N., The Effect of Coagulation Time, Number of Coagulation Bath and Ingredients on Properties of Continuous Graphene Oxide Fiber, Accepted for publication in J. Indian Fibre Tex.

[12] Seyedin S., Romano M.S., Minett A.I., Razal J.M., Sci Rep, 14946 (2015), 1.

[13] Pei S., Cheng H-M., Carbon, 50 (9) (2012), 3210.

[14] Ucar N., Gokceli G., Onen A., Yuksek I.O., KARATEPE YAVUZ N., J. Indian Tex., (2016), 1.

[15] Zhang L., Liang J., Huang Y., Ma Y., Wang Y., Chen Y., Carbon, 47 (2009), 3365.

Received 2017-09-20 Accepted 2018-08-25 